\title{
A survey of the eye complications of leprosy in South Korea
}

\author{
P COURTRIGHT, ${ }^{*}$ R GREEN, R PILARSKI \\ \& J SMUCNY \\ 7113 San Fernando Drive, Boise, Idaho 83704, USA
}

\begin{abstract}
Accepted for publication 2 December 1983
Summary A survey on the ocular complications of leprosy has been carried out in South Korea by members of the American Peace Corps. The results in 2925 patients examined in resettlement villages show the high incidence of ocular damage caused by the disease with over $40 \%$ of the sample having some form of eye problem. As many as $11 \%$ of the patients had visual levels of less than $20 / 200$ in both eyes, and extrapolation of these and other figures emphasizes the magnitude of the problem in the country. The setting up of regional clinics to deal with eye complications of leprosy is recommended.
\end{abstract}

\section{Introduction}

Leprosy has been present in South Korea for many centuries and although sulphones were made generally available 35 years ago the number of affected cases has shown little sign of diminishing. The origins of the disease in the Korean peninsula are uncertain: it may have spread from China in the eighth century AD, or have derived from Japanese invasions in the thirteenth century, but whatever its history, leprosy rapidly became endemic in Korea in the Middle Ages and has remained so ever since.

At present, out of a total South Korean population of about 40 million there are over 28,000 registered patients ${ }^{1}$ and estimates from the Korean Ministry of Health and Social Affairs Department put the figures in the region of 50,000. Field workers in leprosy suggest that numbers may be far higher, perhaps reaching as many as $100-150,000$ cases, ${ }^{2}$ but however accurate the estimates they portray a situation with important and increasing health problems.

A policy of resettlement of leprosy cases has been carried out since 1961 by the

* Reprints: P Courtright. 
South Korean government and the distribution of the patients can be divided on the basis of their location (Figure 1). About 5000 patients (17\%) live in leprosaria some of which are government-sponsored, such as the National Leprosy Hospital on Sorok Island which was started by the Japanese in 1916 and still accommodated over 6000 patients at the end of the Second World War. In addition there are several smaller leprosaria sponsored by Christian churches, such as the Wilson Leprosy Centre-an internationally famous American Presbyterian mission at Suncheon, the St Lazarus Hospital at Shi Hueng and the Sacred Heart Clinic at San Chung. Patients in these leprosaria are generally the chronic severely affected cases, many of whom prefer to remain in these institutions rather than venture out into the community. A high proportion of these patients are disabled and many have advanced eye disease. ${ }^{3}$ The second group of leprosy patients live in resettlement villages of which there are now officially 98 (Figure 2). These

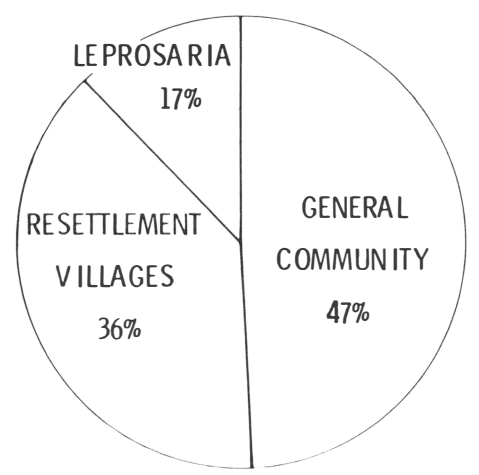

Figure 1. The distribution of registered leprosy patients $(28,298)$ in South Korea, 1981.

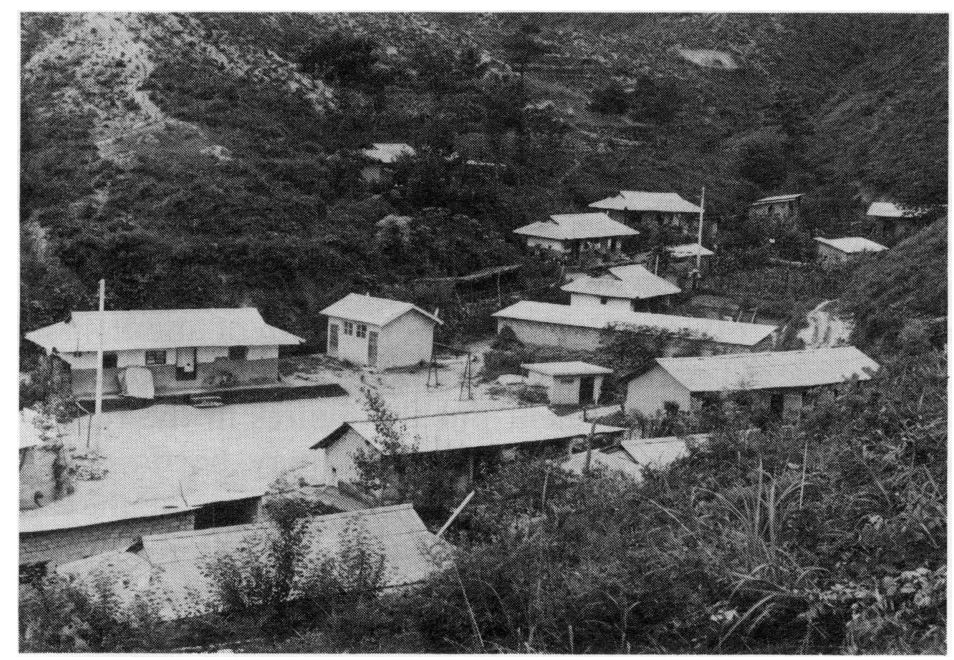

Figure 2. Geang Wha, a typical resettlement village. 
villages are scattered throughout the 8 mainland provinces of South Korea, but are more concentrated in the south of the peninsula (Figure 3). Approximately 10,000 registered leprosy patients $(36 \%)$ live in these villages. The remaining 13,250 registered patients $(47 \%)$ live in the community as do the large numbers not registered with the Health Authority on whom little information is available.

An analysis of the 28,158 registered patients was undertaken in 1979 by the Korean Ministry of Health and Social Affairs and showed that $61 \%$ were male-a male: female ratio of $1 \cdot 5: 1$. The age incidence of these patients is shown in Figure 4 and the type of leprosy was found to be lepromatous in $57 \%$, tuberculoid in $35.6 \%$, borderline in $3.9 \%$ and indeterminate in $3.5 \%$.

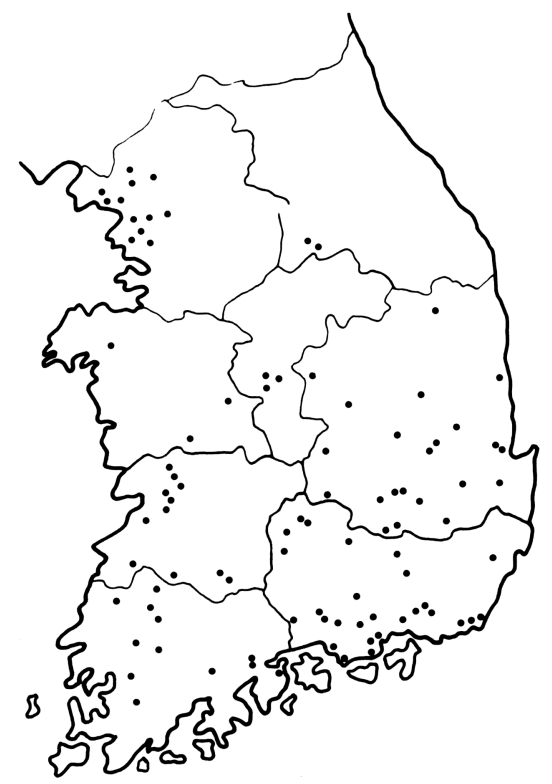

Figure 3. The distribution of 98 resettlement villages in South Korea.

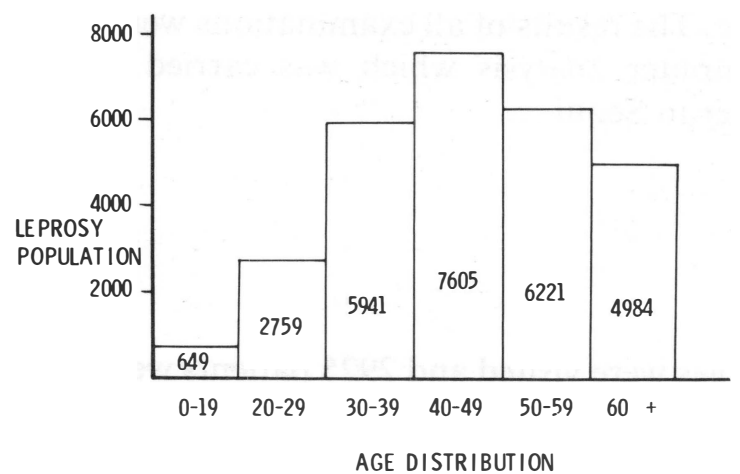

Figure 4. Age distribution of registered leprosy patients in South Korea, 1979. 


\section{Present study}

In 1980 a project on the eye changes associated with leprosy was set up by a group of American Peace Corps workers. The primary objective of this project was to study the eyes of a large number of leprosy patients in the resettlement villages regardless of whether they were experiencing ocular symptoms or not. It was hoped that this would provide statistical information on the prevalence of various types of ocular complication related to leprosy in a country where the disease was common, and by this to identify those patients who were at risk of blindness and who might be helped by surgery that could be carried out at certain hospitals such as the Wilson Leprosy Centre. Prior to the start of the survey the 4 participants in the project had received instructions on methods of eye examination and assessment by members of the staff of the Wilson Leprosy Centre and by visiting ophthalmic surgeons from England.

\section{Procedure}

The selected villages were visited in rotation by members of the survey team working singly or in pairs. Initial information about the villages was obtained from regional health centres. Considerable help was provided by local leprosy workers who accompanied the participants and arranged introductions to village leaders. The name, age and sex of each patient were recorded together with the date of the examination and the name and location of the village. Information on the type, duration and status of leprosy was obtained from the medical records or from the patients themselves. (This data was inadequate in many instances, particularly the details regarding the status of the disease, in which case all patients not definitely known to be positive were recorded as negative.) The basic survey equipment consisted of a Snellen's E test type, a pinhole aperture, a pen torch and in some studies an illuminated loupe for examining the anterior segment of the eye. The results of all examinations were recorded on a proforma designed for computer analysis which was carried out using the American Embassy computer in Seoul.

\section{Results}

A total of 69 villages were visited and 2925 patients were entered into the survey. This represents over two thirds of the resettlement villages in South Korea, with examination of approximately one third of affected individuals. $51 \%$ of the patients were male and the age distribution of both sexes is shown on Figure 5. 


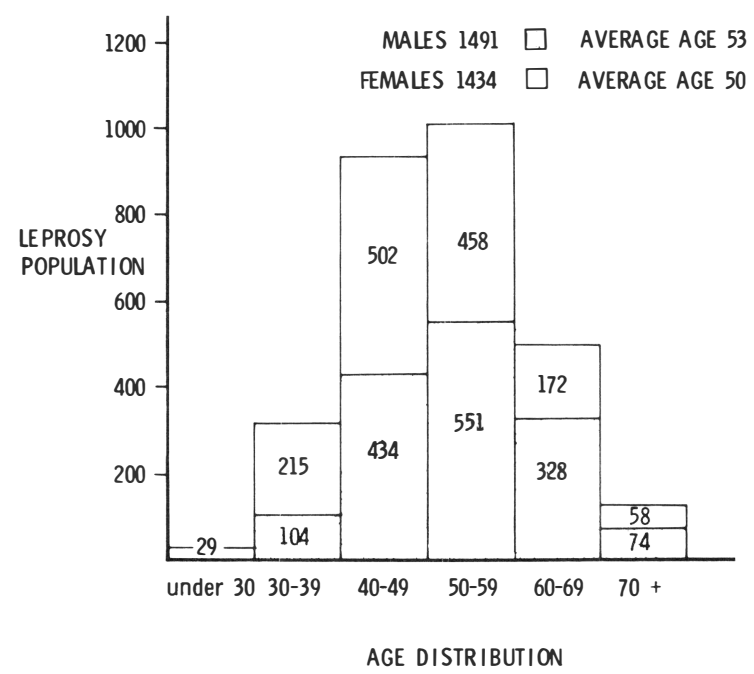

Figure 5. Age distribution of leprosy patients examined.

The type of leprosy was recorded as:

$\begin{array}{lcc} & \text { Male }(\%) & \text { Female }(\%) \\ \text { lepromatous } & 64 & 58 \\ \text { tuberculoid } & 24 & 26 \\ \text { borderline } & 3 & 2 \\ \text { indeterminate } & - & 1 \\ \text { unknown } & 9 & 13\end{array}$

The duration of the disease and the therapy were difficult to assess as information was often unreliable, but most patients had started therapy more than 15 years previously. In only $3 \%$ of the sample was the bacterial index known to be positive.

\section{VISUAL ACUITY}

Ophthalmic examination commenced with measurement of visual acuity with the E test at 20 feet with distance glasses if worn, and patients with vision of less than $20 / 20$ were tested with a pinhole. The results of these measurements are shown on Figure 6 for males and females; of the 5850 eyes examined no significant difference in visual acuity was observed between the right and left eyes. Visual levels of less than 20/200 are considered to represent functional blindness by Western standards and this occurred in $18 \%$ of eyes in male leprosy patients and in $11.5 \%$ of affected females. In $11 \%$ of patients both eyes had vision of less than $20 / 200$, and a total of 251 eyes $(4 \cdot 3 \%)$ in the survey were either absent or had no perception of light (male: female ratio $2 \cdot 5: 1$ ). 

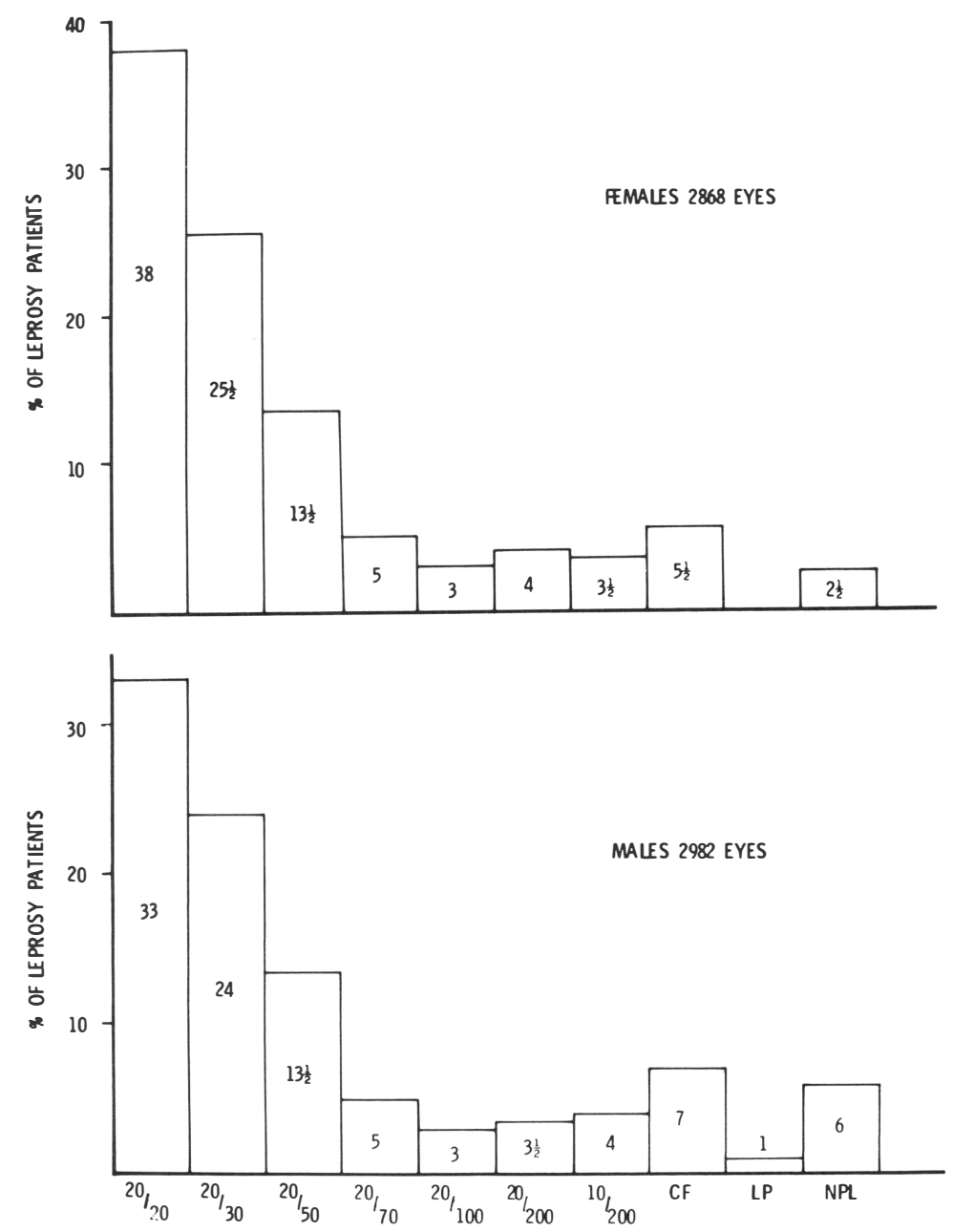

Figure 6. Visual acuity in 2925 leprosy patients examined.

\section{LIDS}

The lids were examined to assess ectropion, lagophthalmos or entropion, and an estimation of lid function was made by asking the patient to close the eyes forcibly against resistance. Severe lagophthalmos, defined as more than $5 \mathrm{~mm}$, was observed in $7 \%$ of eyes and in a further $15 \%$ it was considered to be mild (less than $5 \mathrm{~mm}$ ). Severe ectropion was noted in $2.5 \%$ and in a milder form in $10 \%$, entropion was much less common, affecting only $3 \%$ of eyes examined and associated with trichiasis in $1 \%$. Lid function was therefore found to be affected in at least $25 \%$ of the eyes examined in the sample. 
LACRIMAL APPARATUS

Assessment of the lacrimal function and drainage was not easy to determine but it was possible to identify 14 eyes with inflammation in the region of the lacrimal sac accompanied by symptoms of watering and discharge.

\section{CORNEA}

The cornea was examined with a torch or illuminated loupe. Opacities were graded according to their probable influence on sight and were observed in $11 \%$ of all eyes examined. In half of these cases the opacities were considered to be sufficient to cause significant visual impairment. Blinding opacities were seen more commonly in male patients with a male:female ratio of $2 \cdot 5: 1$. Pterygium was noted in $14 \%$ of eyes with the sexes equally affected. Corneal sensation was tested with cotton wool and in $14 \%$ of males and in $9.5 \%$ of females it was considered to be reduced; in a further $9.5 \%$ of males and $7 \%$ of females sensation was totally absent. Approximately $20 \%$ of the total sample of patients therefore were found to have some impairment of corneal sensation and blinding corneal opacities were seen in over $5 \%$.

IR IS

Examination of the pupils was carried out as much as possible under standard conditions and pupil reactions were tested with a pen torch. By this method an assessment could be made of the size and shape of the pupil and abnormalities of position were noted. Synechiae were seen in some eyes but if small were often difficult to detect. In many cases with advanced corneal disease the pupil abnormalities could not be determined and in several patients previous intra-ocular surgery had altered the pupil shape. In $19.5 \%$ of all eyes in which examination was possible the pupil size was noted to be less than $2 \mathrm{~mm}$ (male:female ratio $1.4: 1$ ) and non-surgical irregularities of pupil shape or position were observed in $8 \%$. The pupil reactions to light were completely absent in $12.5 \%$ of eyes (male:female ratio $1 \cdot 6: 1$ ) and sluggish in a further $17.5 \%$. Synechiae were positively identified in $7 \%$ and 88 eyes had had iridectomies. Pupil abnormalities of some sort were therefore observed in at least $20 \%$ of the total sample, and since in many cases advanced corneal disease prevented observations of the pupil or iris, the proportion of cases with pupil abnormalities is likely to be nearer $25 \%$.

\section{CATARACT}

A slit-lamp or an ophthalmoscope were not available so that it was not easy to determine the presence of lens opacities, especially in eyes with very small pupils. 
Advanced cataract was observed in approximately $5 \%$ of eyes although it is realized clearly that this figure is likely to underestimate the true incidence.

\section{Discussion}

It is evident, both from personal experience and from discussion with leprosy workers in South Korea, that ocular problems make up a high proportion of the out-patient complaints of leprosy sufferers. This survey was designed to give some indication of the incidence of eye complications throughout the leprosy population of South Korea by examining a large number of patients in resettlement villages regardless of whether or not they had ocular symptoms.

The results confirm the suspicion of the high incidence of ocular complications of leprosy-out of a total of 2925 cases examined in the survey at least $40 \%$ had some form of ocular disturbance attributable to the disease. More important was the observation that serious eye complications occurred in a significant percentage of cases - abnormalities of lid function in $25 \%$, impaired corneal sensation in $20 \%$, corneal opacities in $11 \%$ and iris changes in $25 \%$, and in that most of these might be considered to predispose to blindness. Most important of all was the finding that visual acuity measurements showed that $11 \%$ of all patients had vision of less than 20/200 in both eyes and were therefore registerable as blind by Western standards, and that $4.3 \%$ of eyes examined were absent or had no perception of light.

The implications of these findings must be considered in relationship to the total leprosy population in South Korea. If the figures were repeated throughout the country with its 28,000 registered patients at least 3000 of these would be expected to be blind and the numbers are probably considerably higher since they do not take into account the large proportion of unregistered patients, and also because it is known that the cases living in leprosaria (about 5000) have a much higher incidence of advanced eye disease and these were not included in this present survey. Allowing for these factors it is estimated that there are at least 5000 blind leprosy patients living in South Korea and this represents a considerable socio-economic problem.

The difficulties faced by leprosy sufferers because of their loss of sensation and limb deformities are severe enough; if loss or impairment of vision is added to these, the problems become magnified and the implications for the individual and for the social services are obvious. The present care of the ocular problems of leprosy patients in South Korea is limited, with much of the burden being borne by Church missions whose resources are low and already stretched by their work in other fields of leprosy, such as general health care, orthopaedic surgery and dermatology. Many advanced cases need surgery to restore sight or to prevent the onset of blindness, and procedures such as tarsorrhaphy, iridectomy and cataract extraction, although carried out on an intermittent basis at certain centres,${ }^{4}$ are not generally available. 
The main problems which predispose to the more severe ocular complications need routine supervision, relatively simple therapy, and the setting up of out-patient centres equipped for eye examination, situated in easily accessible areas with an ophthalmologist in attendance. Such clinics could be available for regular eye checks on leprosy patients, with instructions being given on basic eye care and preventive measures, and those cases requiring more sophisticated forms of therapy or surgery could be referred to the appropriate centres.

It is hoped that this survey will stimulate further improvement in the care of these patients.

\section{Acknowledgments}

None of this work would have been possible without the help and advice of Dr Yu Gyeong Un and Dr Mia Topple of the Wilson Leprosy Centre, Suncheon, Mr Lee Bong Nae of the Jinju Skin Clinic and Miss Emma Freisinger of the Emma Clinic. We are indebted also to Mr James E H Mayer, Director of the Peace Corps in South Korea, Mr Allen Kellogg of the USA Embassy Seoul and to Mr Timothy ffytche and Mr Malcolm Kerr-Muir, visiting eye surgeons from London who assisted in implementing the project and the preparation of this paper. We are grateful also to the Department of Medical Illustration, St Thomas' Hospital, London and to Miss Gillian McCansh for secretarial assistance. Statistics for registered leprosy patients in South Korea in 1979 and 1981 were supplied by the Korean Ministry of Health and Social Affairs.

\section{References}

1 World Atlas of Leprosy. Publ. Les Amis du Père Damien. 1979.

2 Holmes WJ. Leprosy of the eye in South Korea. Int J Lepr, 1957; 25: 99-103.

3 Rock R, Obara A, Amundsen AM. Report of an ophthalmological survey of Colony patients at the Wilson Leprosy Centre. 1973.

${ }^{4}$ ffytche TJ. Cataract surgery in the management of late complications of lepromatous leprosy in South Korea. Brit J Ophthalmol, 1981; 65: 243-8. 